\title{
Food and Nutrient Intake among People Following Major Upper Gastrointestinal Surgery
}

\author{
Sharon Carey ${ }^{1 *}$, Jane Young ${ }^{2}$, Margaret Allman-Farinelli ${ }^{3}$ \\ ${ }^{1}$ Department of Nutrition and Dietetics, Royal Prince Alfred Hospital, Sydney, Australia; ${ }^{2}$ School of Public Health, University of \\ Sydney, Sydney, Australia; ${ }^{3}$ School of Molecular Biosciences, University of Sydney, Sydney, Australia. \\ Email: *sharon.carey@sswahs.nsw.gov.au
}

Received May $2^{\text {nd }}, 2012$; revised June $2^{\text {nd }}, 2012$; accepted June $9^{\text {th }}, 2012$

\begin{abstract}
Introduction: Surgery to the upper gastrointestinal region of the gut results in symptoms greatly impacting on dietary intake, and a diet high in energy and protein is encouraged. The aim of this study was to examine the food and nutrient intakes of people having had major upper gastrointestinal surgery, and compare them to current food and nutrient recommendations. Materials and Methods: People having had major upper gastrointestinal roux-en-y surgery greater than 6 months ago were recruited. Each person completed a three day food diary and food intakes were compared to the Healthy Food Guide for Australians. Macro and micro-nutrient intakes were compared to the Estimated Average Requirement reference values for Australia and New Zealand. Results: Thirty people were recruited and analysis of dietary intakes indicated inadequate serves of cereals, vegetables, fruit and dairy products compared to recommendations, resulting in below Estimated Average Requirements for many vitamins and minerals. Severely malnourished people, and those having had total gastrectomy surgery, were at greatest risk of not meeting macro and micro-nutrient recommendations. Conclusions: People having had major upper gastrointestinal surgery are encouraged to have a diet high in energy and protein. However this advice seems to be followed at the expense of other food groups, leading to low intakes of many micronutrients. Careful monitoring of dietary intakes and signs of nutrient deficiencies should be included as part of routine follow-up for this group of people. Further research is required to determine whether poor dietary intakes result in clinical deficiencies.
\end{abstract}

Keywords: Dietary Intake; Gastrointestinal Surgery

\section{Introduction}

Major upper gastrointestinal surgeries with a roux-en-y procedure result in the removal of key gastrointestinal structures. Without vital structures such as the lower oesophageal sphincter, stomach, pyloric sphincter and upper region of the small intestine, people suffer symptoms such as anorexia, early satiety, reflux and nausea. In oesophagectomy patients, eating-related symptoms such as loss of appetite, reflux, dry mouth, and oesophageal pain have been reported as the most common and persistent [1]. These symptoms greatly inhibit appetite and oral intake, making it difficult to maintain a healthy nutritional status [2].

Furthermore, altered gut motility resulting in malabsorption, and surgery that removes or bypasses structures essential for absorption, results in a higher risk of deficiency [3].

While standard dietary advice following surgery encourages a calorie and protein rich diet with frequent

"Corresponding author. small meals [4], few researchers have assessed the nutritional adequacy of food consumed by this population. Total calorie and protein intakes appear to be adequate in the small number of studies that have reported such data [5-7]. Dietary intake of vitamins and minerals is also not well documented, and there is little data on key nutrients of concern which include vitamin D [5,6], selenium [6], and calcium $[5,8]$.

The nutritional status of this population has been assessed and found to be compromised compared to the general population [9]. Hence with a high degree of symptom reporting and a compromised nutritional status, nutritional follow-up, dietary assessment and counselling would be essential following such surgery.

This study aimed to assess the dietary intakes of people who had undergone major upper gastrointestinal rouxen-y surgery, compare food and nutrient intakes across surgical and nutritional groups; and to compare food and nutrient intakes to the Healthy Food Guide for Australia [10] and the Estimated Average Requirement (EAR) ref- 
erence values, as set out by the Australian National Health and Medical Research Council [11].

\section{Materials and Methods}

\subsection{Subjects}

Consecutive patients having had major upper gastrointestinal surgery with roux-en-y reconstruction greater than six months ago attending three upper gastrointestinal surgeons' clinics were invited to participate in this study. The clinics covered surgery from three major hospitals in the Sydney region of New South Wales, Australia. Initial interest for study participation was confirmed by the consulting surgeon before the participants were contacted by the research dietitian. Patients with known active disease, inability to consent, or living greater than two hours from the centre were excluded from the study. Time period for recruitment included August 2009 to December 2009 from Royal Prince Alfred Hospital, and from May 2010 to August 2010 from Bankstown and Concord Repatriation General Hospital. Two additional people were recruited from an educational evening for people who had been treated for upper gastrointestinal cancer. This study was approved by the ethics committees at all three health services and the university.

\subsection{Data Collection}

Basic demographic and nutrition assessment data was collected. Nutritional assessment included weight and height, calculation of Body Mass Index and Subjective Global Assessment (SGA) [12].

Nutritional intake was assessed using a 3 day food diary, using household measures of weights and fluids [13]. Participants were asked to record two weekdays and one weekend day, and standard instructions were sent to subjects to facilitate accurate recordings. If people had limited written English skills, a detailed diet history was obtained by the research dietitian instead.

Food Diaries were analysed using the computerized nutrition package Foodworks 2007 (Xyris Software Pty Ltd., Brisbane, Australia). Dietary analysis included measures of macronutrients and micronutrients.

Nutritional requirements for energy were calculated using the Schofield equation for estimating energy requirements [14], where basal requirements were multiplied by 1.5 - 1.8 activity factor [15], dependent on reported level of activity. Protein requirements were based on standard requirements for free living individuals, with a range of 0.8 to $1.0 \mathrm{~g} / \mathrm{kg}$ per day. Energy and protein requirements were calculated for maintenance of current weight, and weights were adjusted for those with a BMI $\geq 30 \mathrm{~kg} / \mathrm{m}^{2}$, in which an adjusted weight half way between ideal and actual weight $[16,17]$ was used to calcu- late requirements. Energy requirements were calculated in kilojoules and protein in grams. To assess for underreporting of dietary intake, the Goldberg "cut-off 1" equation was used [18].

Number of serves per food group were calculated using recommended daily serve sizes [10], and number of people not meeting recommended intakes of the five food groups (cereals, vegetables, fruit, dairy products, meats) was determined. Nutrients were compared to the EAR for Australia and New Zealand, and expressed as a percentage of the recommended nutrient value. Where more than $2 \%$ of cases fell below the EAR, nutritional inadequacy was assumed, as outlined in the EAR cutpoint method [19].

\subsection{Statistical Analysis}

Descriptive statistics were expressed as mean and standard deviation. The Statistical Package for Social Sciences version 18 (SPSS Inc., Chicago, IL) was used for statistical analysis. Differences between surgical and nutritional groups were analyzed using unpaired Student's t-test and univariate analysis of variance (ANOVA), with Tukey's post hoc analysis. Differences between the two dietary assessment methods, and also between normal and under-reporters, were also assessed using unpaired t-test. Statistical significance of $\mathrm{p}<0.05$ was assumed.

\section{Results}

Thirty people (20 males, 10 females) were recruited for this study. The mean age of the group was $67.9( \pm 13.3)$ years and mean time since having surgery was 32.2 $( \pm 26.0)$ months. Types of surgery included total gastrectomy $(\mathrm{n}=5)$, oesophagectomy $(\mathrm{n}=8)$, pancreaticoduodenectomy (Whipple's) procedure $(\mathrm{n}=9)$ and partial/subtotal gastrectomy $(n=8)$. One participant had surgery for severe achalasia; the remainder all had surgery due to neoplasm. No patient received pre-operative nutrition assessment or a standardized nutrition care pathway postoperatively. Five people reported readmission post surgery for nutrition support; three receiving enteral and two receiving parenteral nutrition. At the time of data collection only one participant was having regular monitoring by a dietitian.

Overall nutritional status of the group has been reported elsewhere [9]. Fourteen people displayed some degree of malnutrition on SGA scores, with 10 classified as "mild-to-moderately malnourished" (SGA B) and 4 classified "severely malnourished" (SGA C).

Ten people were unable to provide 3-day food diaries due to limited written English, and detailed diet histories were used to assess dietary intake. No significant differences in nutrient intakes were found between people 
reporting 3-day food diaries versus those using detailed diet histories. The Goldberg "cut-off 1" method equation was used to assess for under-reporting. Eighteen participants showed a degree of under-reporting. As the only significant differences between under-reporters and normal reporters in nutrient intakes were for carbohydrate $(\mathrm{p}$ $=0.05)$ and sodium $(p=0.05)$ intakes, all data was considered appropriate for inclusion in the analysis.

For this group of subjects, mean total energy intake was $7209.5 \pm 2093.5$ kilojoules, compared to estimated energy requirements of $9277.8 \pm 2068.1$ kilojoules. Total energy was derived from carbohydrate $(44.3 \%)$, fat $(35.1 \%)$, protein $(18.1 \%)$ and alcohol $(2.5 \%)$. In comparison with the recommended serves from each of the five food groups, in the Healthy Eating Guide for Australians, this group did not consume adequate cereals, vegetables, fruit or dairy foods, as seen in Table 1. Dietary intake of nutrients and comparison with the EAR can be seen in Table 2. Based on the EAR cut-point method, intakes of protein, thiamin, riboflavin, niacin, vitamin $\mathrm{C}$, folate,

Table 1. People not meeting daily recommended serves according to the Australian Healthy Eating Guide.

\begin{tabular}{|c|c|c|c|c|}
\hline \multirow{2}{*}{ Food group } & \multirow{2}{*}{$\begin{array}{l}\text { Recommended } \\
\text { serving/day }\end{array}$} & \multicolumn{2}{|c|}{$\begin{array}{l}\text { No. of serves/day } \\
\quad \text { Mean }( \pm \mathrm{SD})\end{array}$} & \multirow{2}{*}{$\begin{array}{l}\text { Total number of people not meeting } \\
\text { recommended serves/day }\end{array}$} \\
\hline & & $\begin{array}{l}\text { 3-day food records } \\
\qquad(\mathrm{n}=20)\end{array}$ & $\begin{array}{l}\text { Detailed diet history } \\
\qquad(\mathrm{n}=10)\end{array}$ & \\
\hline Cereals (including breads, rice, pasta, noodles) & $4-12$ & $3.8( \pm 1.9)$ & $3.1( \pm 1.1)$ & 17 \\
\hline Vegetables and legumes & 5 & $3.7( \pm 1.2)$ & $3.4( \pm 1.7)$ & 23 \\
\hline Fruit (including fruit juices) & 2 & $1.9( \pm 1.4)$ & $1.6( \pm 1.2)$ & 14 \\
\hline Milk, yoghurt, cheese & 2 & $1.7( \pm 1.0)$ & $2.2( \pm 2.9)$ & 16 \\
\hline Lean meat, fish, poultry and nuts & 1 & $3.1( \pm 1.4)$ & $2.5( \pm 1.1)$ & 0 \\
\hline Extra foods (fats, oils, sugar, sweets and snacks) & $0-3$ & $5.3( \pm 2.9)$ & $5.1( \pm 3.1)$ & 0 \\
\hline
\end{tabular}

No significant differences between 3 -day food record and diet history data.

Table 2. Reported nutrient intakes compared to Estimated Average Requirements.

\begin{tabular}{|c|c|c|c|}
\hline \multirow{2}{*}{ Nutrient } & \multicolumn{2}{|c|}{ Mean intake ( \pm Standard Deviation) } & \multirow{2}{*}{ Total $\%$ cases below Estimated Average Requirement (EAR) } \\
\hline & 3-day food records $(n=20)$ & Detailed diet history $(\mathrm{n}=10)$ & \\
\hline Energy (KJ) & $7578.8 \pm 2297.9$ & $6471.1 \pm 1437.0$ & \\
\hline Protein $(\mathrm{g})$ & $77.0 \pm 25.2$ & $68.8 \pm 17.9$ & $13 \%$ \\
\hline Total Fat (g) & $75.0 \pm 23.9$ & $54.6 \pm 19.4$ & \\
\hline Cholesterol (g) & $365.4 \pm 188.6$ & $238.7 \pm 70.7$ & \\
\hline Carbohydrate (g) & $186.9 \pm 74.6$ & $177.3 \pm 49.3^{*}$ & \\
\hline Fibre (g) & $17.1 \pm 6.9$ & $16.7 \pm 5.1$ & (a) \\
\hline Thiamin (mg) & $1.7 \pm 1.1$ & $3.6 \pm 3.9^{*}$ & $20 \%$ \\
\hline Riboflavin (mg) & $2.0 \pm 0.6$ & $3.6 \pm 4.1^{*}$ & $23 \%$ \\
\hline Niacin Eqv (mg) & $34.4 \pm 14.9$ & $31.8 \pm 9.1$ & $3 \%$ \\
\hline Vitamin C (mg) & $100.0 \pm 76.5$ & $123.5 \pm 91.5$ & $7 \%$ \\
\hline Folate $(\mu \mathrm{g})$ & $244.1 \pm 91.6$ & $202.2 \pm 74.1$ & $80 \%$ \\
\hline Vitamin $\mathrm{A}(\mu \mathrm{g})$ & $1004.0 \pm 390.4$ & $1476.1 \pm 2227.2^{*}$ & $20 \%$ \\
\hline Potassium (mg) & $2125.8 \pm 843.9$ & $1819.3 \pm 428.3$ & (b) \\
\hline Sodium (mg) & $2563.3 \pm 941.5$ & $2588.1 \pm 900.7$ & (c) \\
\hline Magnesium (mg) & $258.1 \pm 92.7$ & $316.4 \pm 161.1^{*}$ & $87 \%$ \\
\hline Calcium (mg) & $794.0 \pm 249.6$ & $668.8 \pm 611.4^{*}$ & $73 \%$ \\
\hline Phosphorus (mg) & $1299.0 \pm 344.0$ & $1145.6 \pm 439.2$ & $3 \%$ \\
\hline Iron (mg) & $10.3 \pm 3.8$ & $11.0 \pm 3.4$ & $3 \%$ \\
\hline Zinc (mg) & $9.1 \pm 2.9$ & $11.6 \pm 4.5$ & $63 \%$ \\
\hline
\end{tabular}

*Significant difference between the two groups ( $\mathrm{p}>0.05$ ); (a) Adequate intake level for dietary fibre is $30 \mathrm{~g}$ for males and $25 \mathrm{~g}$ for females; (b) Adequate intake level for potassium is $3800 \mathrm{mg}$ for males and $2800 \mathrm{mg}$ for females; (c) Adequate intake level for sodium is $460 \mathrm{mg}$ for males and $420 \mathrm{mg}$ for females. 
vitamin A, magnesium, calcium, phosphorus, iron and zinc were inadequate.

Univariate analysis (ANOVA) was used to assess differences in dietary intake across nutritional groups and the different surgical procedures. People with severe malnutrition (SGA C) had significantly lower total fat intake compared to well nourished (SGA A) people ( $\mathrm{p}=$ $0.041)$; and consumed significantly less meat $(p=0.017)$ and dairy food $(p=0.000)$. Severely malnourished (SGA C) people also consumed significantly less dairy food compared to mild/moderately malnourished (SGA B) people $(p=0.000)$. People who had a total gastrectomy reported a dietary intake significantly lower in protein $(\mathrm{p}$ $=0.047)$, total fat $(p=0.016)$, saturated fat $(p=0.042)$, monounsaturated fat $(p=0.017)$, calcium $(p=0.039)$ and phosphorus $(\mathrm{p}=0.049)$ when compared to people who had pancreaticoduodenectomy. They also consumed significantly less meat $(\mathrm{p}=0.024)$ and vegetables $(\mathrm{p}=$ $0.011)$.

\section{Discussion}

Major upper gastrointestinal tract surgery has a profound impact on the nutritional intake and absorption of both macro and micronutrients. Dietary advice following such surgery usually encourages the inclusion of high energy and protein foods due to early satiety [4]. Higher intakes of meats, dairy products and other high energy snacks is encouraged. However, it is still important to ensure people consume a balanced diet, including cereals, vegetables and fruits to meet macro and micro-nutrient requirements. While this study group had macronutrient intakes higher in fat and protein than the recommended "healthy diet", the main contributors were meat products and "extra" (energy-dense nutrient-poor) foods. Consumption of dairy products was not increased, and half the group did not meet the recommendation of two serves of dairy products per day.

Furthermore, low intakes of cereals, vegetables and fruits have resulted in a large shortfall in many micronutrients. Folate and magnesium are nutrients predominately found in the above food groups. The low intake from these food groups is reflected in the high number of people not meeting EAR for these nutrients. Cereals and dairy products also contribute significant quantities of zinc in a normal diet, making this a nutrient of concern, despite the higher meat consumption.

While it is known that the nutrient intakes of the healthy Australian population do not meet the EAR for many micronutrients [20], this is of much greater concern for people having had major upper GI surgery as they are already at increased risk of micronutrient deficiencies due to altered digestion and absorption. Calcium intake has previously been highlighted as a nutrient of concern [5], and the current findings support this misgiving. More than half of this study group failed to consume the recommended intake for dairy products, and were not meeting the EAR for calcium. Historically bone mineral disease has been prevalent in post-gastrectomy [21] and continues to be a complication well documented $[22,23]$. Vitamin D, selenium and Vitamin $B_{12}$ were not able to be assessed using the current computerized nutrition package but have previously been nutrients of concern. Findings from this study indicate that there are many nutrients at risk. The current study strongly supports more detailed research to investigate whether the low intake of micronutrients translate into serum and/or clinical deficiencies.

While immediate nutritional advice for this group is focused on minimizing weight loss, the role of the dietitian should continue into recovery to ensure a balanced dietary intake meeting all nutrient requirements. Nutritional monitoring should include biochemical and clinical assessment to detect macro and micro-nutrient deficiencies. In a recent survey of dietitians across Australia, it was found that there was no structured nutritional followup for this group of patients, with only a small number receiving dietetic input after hospital discharge [24]. When patients are reviewed post discharge, vitamin and mineral levels are rarely checked. This is of major concern, particularly for those people who are severely malnourished, and those having had total gastrectomy surgery, which appear to be at greatest risk. For those people that have ongoing malnutrition, vitamin and mineral supplementation should also be considered.

The limitations of analyzing dietary intakes are well documented $[25,26]$. Using detailed diet histories alongside 3 day diet diaries introduces a methodological inconsistency. However, upper GI cancers are more prevalent in some Asian and European groups [27], resulting in a group of patients with limited written English whose exclusion would create significant bias. For this reason both sets of data were included in the final analysis.

While there is limited research assessing the long term nutritional status of people who have had major upper GI surgery, the current study indicates that the dietary intake of this group is compromised. Further research is required to assess the clinical impact of such a finding, and provide a pathway for nutritional management.

\section{REFERENCES}

[1] P. Viklund, Y. Wengström, I. Rouvelas, M. Lindblad and J. Lagergren, "Quality of Life and Persisting Symptoms after Oesophageal Cancer Surgery," European Journal of Cancer, Vol. 42, No. 10, 2006, pp. 1407-1414.

doi:10.1016/i.ejca.2006.02.005

[2] G. Bovio, G. Montagna, C. Bariani and P. Baiardi, "Upper Gastrointestinal Symptoms in Patients with Advanced Cancer: Relationship to Nutritional and Performance Status," Supportive Care in Cancer, Vol. 17, No. 10, 2009, 


\section{pp. 1317-1324. doi:10.1007/s00520-009-0590-x}

[3] J. Scholmerich, "Postgastrectomy Syndromes-Diagnosis and Treatment," Best Practice \& Research in Clinical Gastroenterology, Vol. 18, No. 5, 2004, pp. 917-933.

[4] B. Thomas, Ed., "Manual of Dietetic Practice," 4th Edition, Blackwell Science, Oxford, 2007.

[5] S. Bisballe, S. Buus, B. Lund and I. Hessov, "Food Intake and Nutritional Status after Gastrectomy," Human Nutrition-Clinical Nutrition, Vol. 40, No. 4, 1986, pp. 301308.

[6] C. Maskell, P. Daniels and C. D. Johnson, "Dietary Intake after Pancreatectomy," British Journal of Surgery, Vol. 86, No. 3, 1999, pp. 323-326. doi:10.1046/j.1365-2168.1999.01033.x

[7] D. J. Ludwig, R. C. Thirlby and D. E. Low, "A Prospective Evaluation of Dietary Status and Symptoms after Near-Total Esophagectomy without Gastric Emptying Procedure," American Journal of Surgery, Vol. 181, No. 5, 2001, pp. 454-458. doi:10.1016/S0002-9610(01)00600-6

[8] E. B. Marcinowska-Suchowierska, M. J. Talalaj, A. W. Wlodarcyzk, K. Bielecki, J. J. Zawadzki and R. Brzozowski, "Calcium/Phosphate/Vitamin D Homeostasis and Bone Mass in Patients after Gastrectomy, Vagotomy, and Cholecystectomy," World Journal of Surgery, Vol. 19, No. 4, 1995, pp. 597-601. doi:10.1007/BF00294730

[9] S. Carey, D. Storey, A. V. Biankin, D. Martin, J. Young and M. Allman-Farinelli, "Long Term Nutritional Status and Quality of Life Following Major Upper Gastrointestinal Surgery-A Cross-Sectional Study," Clinical Nutrition, Vol. 30, No. 6, 2011, pp. 774-779.

[10] Australian Government Department of Health and Aging, "Australian Guide to Healthy Eating," Australian Government, Department of Health and Aging, Canberra, 1998.

[11] Australian Government Department of Health and Aging, National Health and Medical Research Council, "Nutrient Reference Values for Australian and New Zealand Executive Summary," Commonwealth of Australia, Canberra, 2006.

[12] A. Detsky, J. McLaughlin and J. Baker, "What Is Subjective Global Assessment of Nutritional Status?" Journal of Parenteral and Enteral Nutrition, Vol. 11, No. 1, 1987, pp. 8-13. doi:10.1177/014860718701100108

[13] R. Gibson, "Principles of Nutrition Assessment," 2nd Edition, Oxford University Press, New York, 2005.

[14] W. N. Schofield, "Predicting Basal Metabolic Rate, New Standards and Review of Previous Work," Human Nutrition-Clinical Nutrition, Vol. 39, Suppl. 1, 1985, pp. 541.

[15] World Health Organisation, Energy and Protein Requirements, "Report of a Joint FAO/WHO/UNU Expert Consultation," World Health Organization Technical Report
Series, No. 724, 1985, pp. 1-206.

[16] N. Barak, E. Wall-Alonso and M. D. Sitrin, "Evaluation of Stress Factors and Body Weight Adjustments Currently Used to Estimate Energy Expenditure in Hospitalized Patients," Journal of Parenteral \& Enteral Nutrition, Vol. 26, No. 4, 2002, pp. 231-238. doi: $10.1177 / 0148607102026004231$

[17] S. Ferrie and M. Ward, "Back to Basics: Estimating Energy Requirements for Adult Hospital Patients," Nutrition and Dietetics, Vol. 64, No. 3, 2007, pp. 192-199. doi:10.1111/j.1747-0080.2007.00124.X

[18] G. R. Goldberg, A. E. Black, S. A. Jebb, T. J. Cole, P. R. Murgatroyd, W. A. Coward and A. M. Prentice, "Critical Evaluation of Energy Intake Data Using Fundamental Principles of Energy Physiology: 1. Derivation of Cut-Off Limits to Identify Under-Reporting," European Journal of Clinical Nutrition, Vol. 45, No. 12, 1991, pp. 569-581.

[19] S. P. Murphy and M. I. Poos, "Dietary Reference Intakes: Summary of Applications in Dietary Assessment," Public Health Nutrition, Vol. 5, No. 6, 2002, pp. 843-849. doi:10.1079/PHN2002389

[20] W. McLennan and A. Podger, "1995 National Nutrition Survey: Nutrient Intakes and Physical Measurements," Australian Bureau of Statistics, Canberra, 1998.

[21] C. Clark and J. Crooks, "Osteomalacia after Gastrectomy," The Lancet, Vol. 2, No. 7426, 1965, pp. 1347-1348. doi:10.1016/S0140-6736(65)92370-6

[22] American Gastroenterological Association Medical Position Statement, "Guidelines on Osteoporosis in Gastrointestinal Diseases," Gastroenterology, Vol. 124, No. 3, 2003, pp. 791-794. doi:10.1053/gast.2003.50107

[23] K. H. Baek, H. M. Jeon, S. S. Lee, D. J. Lim, K. W. Oh, W. Y. Lee, et al., "Short-Term Changes in Bone and Mineral Metabolism Following Gastrectomy in Gastric Cancer Patients," Bone, Vol. 42, No. 1, 2008, pp. 61-67. doi:10.1016/j.bone.2007.08.027

[24] S. Carey, L. He and S. Ferrie, "Nutritional Management of Patients Undergoing Major Upper Gastrointestinal Surgery: A Survey of Current Practice in Australia," Nutrition and Dietetics, Vol. 67, No. 4, 2010, pp. 219-223. doi:10.1111/j.1747-0080.2010.01466.x

[25] I. H. Rutishauser, "Dietary Intake Measurements," Public Health Nutrition, Vol. 8, No. 7A, 2005, pp. 1100-1107. doi:10.1079/PHN2005798

[26] J. M. De Castro, "Eating Behavior: Lessons from the Real World of Humans," Nutrition, Vol. 16, No. 10, 2000, pp. 800-813. doi:10.1016/S0899-9007(00)00414-7

[27] E. Tracey, N. Alam, W. Chen and J. Bishop, "Cancer in New South Wales: Incidence and Mortality Report," Cancer Institute of New South Wales, New South Wales Health, Sydney, 2006. 